\title{
Functional Abdominal Pain in Relation to Behavioral Pattern Triggers in Children
}

\author{
Dhanasekhar Kesavelu ${ }^{\mathrm{a}, \mathrm{b}}$, Keerthi Pai ${ }^{\mathrm{c}}$, Krishna Chaitanya Veligandla ${ }^{\mathrm{d}, \mathrm{e}}$
}

\begin{abstract}
Background: Functional abdominal pain (FAP) in children is a common problem encountered by clinicians. The etiology of FAP is multifactorial and this study explains the correlates of FAP to certain behavioral patterns in children. This is the first Indian study which looks into this area.

Methods: Eighteen participants between 4 and 18 years old who had consulted pediatric gastroenterologist with abdominal pain in one single multi-specialty hospital were included. A written consent was obtained from parents. The Child Behavior Checklist (CBCL) was given to the parents to understand the various behavioral problems in the children; and intelligence quotient (IQ) assessments were done in all children. A 5-point Likert scale was used to assess anxiety in children which was rated by the child.
\end{abstract}

Results: The CBCL identified the stressors reported by children such as academic pressure, difficulty to comprehend, need for affection from parents, interpersonal problems at home (frequent arguments between parents, comparison with a brighter sibling) and poor selfesteem. It is observed that $11 / 18(61 \%)$ children had average or above average IQ and 7/18 (39\%) children had low average or dull IQ. The anxiety levels of children in majority, 16 out of 18 children (88.8\%) who presented with FAP, were found to be high or very high on a Likert scale.

Conclusions: Children with FAP have variable IQ levels and high anxiety levels. The need for clinicians to counsel parents and children in order to address the stressors at home and school is warranted as this may help in management of FAP in children. Further multicentric research is necessary to understand these findings in Indian children.

Manuscript submitted April 15, 2020, accepted May 12, 2020

Published online June 18, 2020

aApollo Children's Hospital, Chennai, India

bUniversity of Liverpool, Liverpool, UK

'SS Child Care, Chennai, India

dMedical Affairs, Global Generics India, Dr Reddy's Laboratories Ltd, Hyderabad, India

${ }^{e}$ Corresponding Author: Krishna Chaitanya Veligandla, Medical Affairs, Global Generics India, Dr Reddy's Laboratories Ltd, Hyderabad, India.

Email: krishnacv@drreddys.com

doi: https://doi.org/10.14740/ijcp371
Keywords: Functional abdominal pain; Behavioral patterns; CBCL; IQ

\section{Introduction}

The global prevalence of functional abdominal pain (FAP) disorders is estimated to be $15 \%$ [1]. A recent survey conducted in over 800 Indian school children documented that $92.9 \%$ of recurrent abdominal pain in children is functional in nature [2].

The Rome IV criteria (2016) allow for clinical diagnosis of FAP and related functional conditions (irritable bowel syndrome, functional dyspepsia, and abdominal migraine) [3]. FAP can be diagnosed based on symptoms after appropriate medical evaluation where the symptoms cannot be attributed to another or any other medical condition(s).

The diagnostic Rome IV criteria for FAP must be fulfilled for at least 2 months before diagnosis, must be met at least four times per month, and include all of the following: 1) Episodic or continuous abdominal pain that does not occur solely during physiological events such as eating and menses; 2) Insufficient criteria for other functional gastrointestinal (GI) disorders including irritable bowel syndrome, functional dyspepsia, or abdominal migraine; and 3) After appropriate evaluation, the abdominal pain cannot be fully explained by another medical condition.

It is a known fact that psychological factors play an important role in causation and triggering the onset of FAP in children. Studies have shown positive correlation between anxiety and FAP [4].

Anxiety is a normal human emotion which encompasses behavioral, affective and cognitive responses to situations perceived as uncontrollable or unavoidable. In moderation, anxiety stimulates an anticipatory and adaptive response to challenging or stressful events. In excess, anxiety destabilizes the individual resulting in a dysfunctional state [5].

A meta-analysis was done using 13 psychiatric epidemiological studies with a total sample size of 33,572 subjects who met the criteria; door-to-door survey including all age groups showed the prevalence rate at $20.7 \%(18.7-22.7 \%)$ for all neurotic disorders, which was the highest among all psychiatric disorders [6].

In an urban survey of 109 families for psychiatric morbidity in children below 12 years, Sethi et al [7] found emo- 


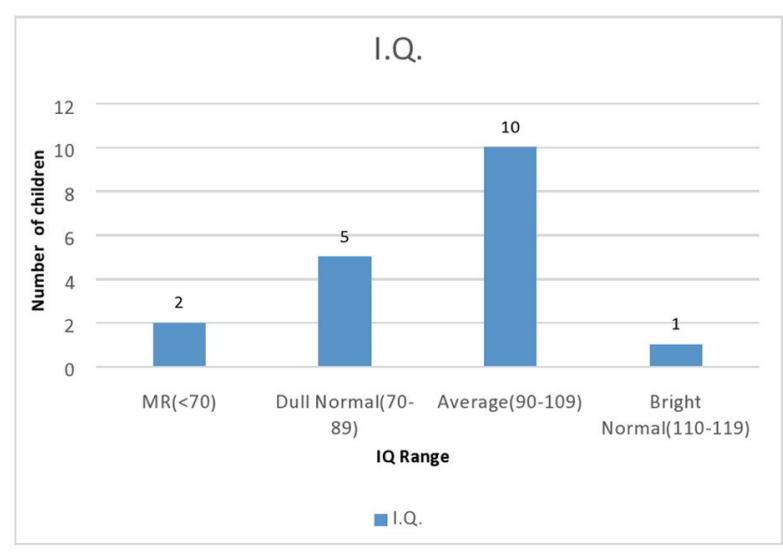

Figure 1. Intelligence quotient of children in analysis.

tional disturbance in $55 \%$ families and $35.4 \%$ in the total children surveyed. The common stressors reported were academic difficulties, family problems, peer problem and sibling rivalry.

Various researches indicate high anxiety in children with FAP, manifesting mainly as nausea, vomiting, loss of appetite and fussy eating patterns. Most of the investigations done were within normal limits for these children. The prevalence of anxiety disorders in children (especially female children) is high in the current situation due to the numerous pressures, mainly from home and school, and the multiple roles a child has to play in today's world. The aim of this study is to ascertain the correlates of functional gastrointestinal disorders (FGIDs) and anxiety in children.

\section{Materials and Methods}

Children who qualified the Rome IV criteria for FAP in whom the clinician, pediatric gastroenterologist excluded organic causes by appropriate blood investigations and imaging (pertaining to individual cases) and endoscopy where appropriate/ indicated and warranted were included in the study.

The definition of FAP in this study is as defined by Rome IV criteria, FAP-not otherwise specified (FAP-NOS) (as mentioned in Introduction). The minimum duration of the symptoms for the children is 2 months as per Rome IV criteria. A clinical psychologist performed the tests conducted in this study. The study has been approved by institutional ethics committee at study site.

Eighteen children with FAP as defined by Rome IV were identified and assessed using intelligence quotient (IQ) test and Likert scale. Children with a known cause or previously diagnosed GI disease such as but not limited to gastro-esophageal reflux disease or inflammatory bowel disease, children with age less than 3 years and children above 17 years, and children whose parents did not given consent were excluded from the study.

A cross sectional survey method was used to collect data from the children and their mothers. All the participants belonged to middle and high socioeconomic status. All the chil-
Table 1. IQ of Children in Analysis

\begin{tabular}{ll}
\hline IQ level & Number of children (n) \\
\hline$<70$ (Mental Retardation) & 2 \\
$70-89$ (Dull Normal) & 5 \\
$90-109$ (Average) & 10 \\
$109-119$ (Normal) & 1 \\
\hline
\end{tabular}

IQ: intelligence quotient.

dren were assessed using the Binet Kamat Intelligence Test (BKT) to assess their IQ. The BKT [8] is a well-researched tool to assess intelligence of children from 3 years to 22 years, and it has norms based on Indian students.

Child Behavior Checklist (CBCL, Achenback 1972) [9] was provided to the parents mostly mothers to obtain information about the child's social, behavioral and emotional problems. A 5-point self-reported Likert scale [10] was given to children to rate their anxiety level with 1 being the lowest and 5 being the highest (1: very low, 2: low, 3 : normal, 4 : high, 5 : very high).

\section{Results}

A total of 18 children diagnosed with FAP completed the study. Twelve out of 18 children affected were female (66.6\%).

IQ

The bar diagram indicates the IQ of the group (Fig. 1) and results are mentioned in Table 1.

It is evident from the bar diagram that majority of the children were of average IQ level: 1) $60 \%$ with average intelligence; 2) 30\% with low average (borderline and dull normal) intelligence; 3) $5 \%$ with mild mental retardation (i.e. IQ below 70 ); and 4) 5\% with above average intelligence (bright normal).

\section{Likert scale}

All the children were provided a 5-point Likert scale to assess their current anxiety. Most of the children, 16 out of 18 children, were rated at high and very high, which indicates that they had insight into their anxiety. Out of 16 children, 10 rated high (4/5) and six rated very high (5/5) anxiety levels. The results are mentioned in Table 2.

\section{CBCL}

It is observed that children (especially women) were "allowed" to somatize their emotional problems rather than directly act it out. Parents were willing to allow the child to stay at home if she had physical symptoms like nausea or vomiting rather deal with anxiety in the child. 
Table 2. Likert Score for Analyzing Anxiety Levels

\begin{tabular}{ll}
\hline Anxiety score \& level & Number of children (n) \\
\hline 5-Very High & 6 \\
4-High & 10 \\
3-Normal & 1 \\
2-Low & 1 \\
1-Very Low & 0 \\
\hline
\end{tabular}

Interestingly the children reported that their anxiety levels would reduce if the stressors were addressed. The most common stressors reported by children were related to academic pressure, difficulty to comprehend, need for affection from parents, interpersonal problems at home (frequent arguments between parents, comparison with a brighter sibling) and poor self-esteem.

\section{Discussion}

Previous studies do indicate a correlation of FAP in children with anxiety. However, most studies have not acknowledged the need for assessing the intelligence and psychosocial stressors [11-13]. Our study has evaluated these parameters.

Our study supports the existing literature that emotional problems were identifiable more in the female (girls) population compared to boys suffering with FAP [14].

In a study conducted in 6 - 13 year old children, $40.7 \%$ of anxious children diagnosed with one or more anxiety disorders had symptoms of an FGID when compared to 5.9\% of nonanxious children with symptoms of an FGID [15]. Conversely in our study, majority of children diagnosed with FAP had high to very high levels of anxiety.

The millennial children have multiple roles to play in school and at home. The quality of life of child is affected owing to undue pressure from parents and school to exceed. In a study conducted by Warschburger et al, children suffering from FGID had lower health-related quality of life (HRQOL) compared to children suffering from organic GI disorders [16].

As is evident from our study, majority $(60 \%)$ of the subject population had normal intelligence. Parents and the child were informed about his/her IQ. It was observed that when the parents and child were told about the child's intellectual functioning they were able to feel less stressed, which may be due to the fact that many a times the child may have got less marks than expected. There is a tendency observed in Indian parents to associate intelligence with academic performance. Usually parents are disappointed when a child gets low marks and all the children expressed their desire to "please their parents with good marks". Parents were less likely to scold/reprimand a child when he/she gets fewer marks when he "is suffering from illness".

The academic pressure on the child was observed to be reduced when the parents knew that the child is intelligent and academic performance is not the only gold standard for intellectual functioning. It is therefore essential that a child present- ing with functional GI symptoms be also assessed for IQ.

Regarding pediatric patients with below average intellectual functioning, it was observed that parents were willing to accept the child's IQ after counseling. This also reduced the stress on the child because the parents reported to be willing to not exert excess pressure on the child and allow him/her to pursue his/her academic interests; this in turn reduces the stress on the child and reduces the functional GI symptoms. Individual interviews with each child highlighted the need for the parents to be sensitive to their psychological problems.

Most of the children in the sample were found to be internalizing the anxiety and were termed as "sick" by the parents; and were avoiding school due to "health problems". Parents especially the mothers were found to be extremely anxious when the child expressed any health problem.

Brief counseling was given to the parents and children focusing primarily on psycho-education and supportive counseling; and the need for the parent to identify the source of stressor and provide supportive methods to the children was emphasized. They were counseled about the need to reduce primary reinforcement of the symptom, which increased the probability of the symptoms and to provide positive reinforcement to the child when he/she exhibited adaptive behaviors like eating healthy food, attending school regularly, playing with friends, and verbalizing his/her anxiety. They were also instructed about not to displace their anger on to the child and sort interpersonal problems between them without involving the child and the children were encouraged to use distraction and coping mechanisms.

The weakness of the study includes a lack of control group and small number of participants in the study.

\section{Conclusions}

The study emphasizes the need for parents to understand that there are various psychological stressors impinging on today's children. Pediatricians and gastroenterologists need to be aware of the prerequisite to sensitize parents about psychological stressors on the child. There is also a strong need for clinical psychologists working with children to liaison with pediatricians and pediatric gastroenterologists. It has been observed that the prognosis is far better when psychosocial stressors were identified and professional counseling was given to both the parent and the child suffering from anxiety.

Future research should focus on correlation of anxiety, intelligence and effect of various psychosocial stressors in GI disorders in children, with a larger cohort and multi-centric approach.

Similarly, research needs to focus on: 1) Long-term effects of psychosocial stressors; 2) Benefits of behavior therapy and parental counseling; 3) Alleviation of GI and other somatic complaints in children; 4) Use quality of life (QOL) score to assess response pre and post therapy; and 5) Role of adjunct drug therapy such as probiotics in the management of FAP.

Parents, medical doctors and clinical psychologists should work in liaison to improve the quality of life of each child. The tests used in study were IQ test (BKT), CBCL and Likert scale. 


\section{Acknowledgments}

The authors are grateful to all participants in the study including children and parents/care givers.

\section{Financial Disclosure}

None to declare.

\section{Conflict of Interest}

None to declare.

\section{Informed Consent}

Informed consent is obtained in the study from all the participants after detailed explanation of study objectives and their role in vernacular language.

\section{Author Contributions}

DK contributed to the idea and design of study, acquisition of data, writing of manuscript, and final approval of manuscript; $\mathrm{KP}$ gathered analyzed data; $\mathrm{KCV}$ analyzed the data and wrote the manuscript.

\section{Data Availability}

The authors declare that data supporting the findings of this study are available within the article.

\section{References}

1. Herzlinger M, Cerezo C. Functional abdominal pain and related syndromes. Child Adolesc Psychiatr Clin N Am. 2018;27(1):15-26.

2. Singh MK, Sinha SK. Etiology of recurrent abdominal pain in children. International Journal of Medical and Health Research. 2017;3(4):147-151.

3. Hyams S, Di Lorenzo C, Saps M, Shulman RJ, Staiano A, van Tilburg M. Functional disorders: children and adoles- cents. Gastroenterology. 2016;150:1456-1468.

4. Newton E, Schosheim A, Patel S, Chitkara DK, van Tilburg MAL. The role of psychological factors in pediatric functional abdominal pain disorders. Neurogastroenterol Motil. 2019;31(6):e13538.

5. Diagnostic and statistical manual for the assessment of mental disorders. 4th ed. Washington, DC: American Psychiatric Association; 1994.

6. Chandrashekhar CR, Reddy MV. Prevalence of mental and behavioral disorders in India: A meta-analysis. Indian J Psychiatry. 1998;40:149-157.

7. Sethi BB, Gupta SC, Kumar R. 300 urban families (a psychiatric study). Indian J Psychiatry. 1967;9:280-302.

8. Sunita Devi. A comparative study on profile analysis of BinetKamat Test of intelligence of children having mild intellectual disability with and without Down syndrome. Eur Acad Res. 2014;II(7).

9. Mazefsky CA, Anderson R, Conner CM, Minshew N. Child behavior checklist scores for school-aged children with autism: preliminary evidence of patterns suggesting the need for referral. J Psychopathol Behav Assess. 2011;33(1):31-37.

10. Saul McLeod. Likert scale definition, examples and analysis. 2019. Accessed at: https://www.simplypsychology. org/likert-scale.html.

11. Math SB, Chandrashekar CR, Bhugra D. Psychiatric epidemiology in India. Indian J Med Res. 2007;126(3):183192.

12. Ganguli HC. Epidemiological findings on prevalence of mental disorders in India. Indian J Psychiatry. 2000;42(1):14-20.

13. Madhav M. Epidemiological study of prevalence of mental disorders in India. Indian J Community Med. 2001;26(4):10-12.

14. Oswari H, Alatas FS, Hegar B, Cheng W, Pramadyani A, Benninga MA, Rajindrajith S. Functional abdominal pain disorders in adolescents in Indonesia and their association with family related stress. BMC Pediatr. 2019;19(1):342.

15. Waters AM, Schilpzand E, Bell C, Walker LS, Baber K. Functional gastrointestinal symptoms in children with anxiety disorders. J Abnorm Child Psychol. 2013;41(1):151163.

16. Warschburger P, Hanig J, Friedt M, Posovszky C, Schier $\mathrm{M}$, Calvano C. Health-related quality of life in children with abdominal pain due to functional or organic gastrointestinal disorders. J Pediatr Psychol. 2014;39(1):4554. 